\title{
Fabrication and Characterization of Hydrogen Sensors Using Graphenes Formed on the 3C-SiC Thin Films
}

\author{
Sang-Jin Bae, Kang-San Kim, Gwiy-Sang Chung* \\ School of electrical engineering, University of Ulsan, Republic of Korea \\ *gschung@ulsan.ac.kr
}

\begin{abstract}
This paper presents the fabrication and characterization of graphene based hydrogen sensors. Graphenes were synthesized by annealing process of $\mathrm{Ni} / 3 \mathrm{C}-\mathrm{SiC}$ thin film. The Ni thin film was deposited on a 3C-SiC substrate and used extracts the substrate's carbon atoms under rapid thermal annealing. Synthesized graphene transferred onto $\mathrm{SiO}_{2}$ substrate by chemically etching of $\mathrm{Ni}$ in $\mathrm{HF}$ solution. Au electrode on the graphene shows ohmic contact and the resistance was changed with hydrogen concentration. Initial resistance of graphene was around of $360 \Omega$ and Pd coated graphene resistance shows $27 \Omega$. Sensing performance of hydrogen sensors based on the graphene is improved with Pd catalyst. The response factors of graphene and $\mathrm{Pd}$ on graphene are 0.28 and $1.26 \%$, respectively, at 50 ppm hydrogen concentration.
\end{abstract}

Key words: Graphene, 3C-SiC thin film, Hydrogen sensor, Transferred, Pd catalyst

\section{Introduction}

Graphene, a potential candidate, after carbon nanotubes, for use in future nanodevices, has attracted wide attention since its discovery in 2004 [1]. However, the some particular characteristics of graphene make it possible to increase the sensitivity to its ultimate limit and detect individual gas macular. First, graphene is a strictly two-dimensional material and, as such, has its whole volume exposed to surface as adsorbents, which maximizes their effect. Second, graphene is highly conductive, exhibiting metallic conductivity and, hence, low Johnson noise even in the limit of no charge carriers [2]. Third, graphene has few crystal defects, which ensure a low level of excess noise caused by their thermal switching [3]. All these features contribute to make a unique combination that maximizes the signal-to-noise ratio to a level sufficient for detecting changes in a local concentration by less than one electron charge $e$ at room temperature. However, large-area single-layer graphenes are hard to obtain via the adhesive tape, mechanical cleavage and chemical exfoliation methods. On the other hand, the growth of epitaxial graphene (EG) possesses large single-crystalline domains with uniform thickness using silicon carbide substrates. However, this structure is difficult to transfer EG from SiC to another substrate. Thus transition metals $(\mathrm{Cu}, \mathrm{Ru}$ and $\mathrm{Ni}$ ) are employed for easily transfer onto the specific substrate.
In this work, Ni/3C-SiC structure was used for synthesis of graphene by rapid thermal annealing. After remove the Ni layer in HF for $10 \mathrm{~min}$, graphene transferred onto the $\mathrm{SiO}_{2}$ for apply to resistivity type hydrogen sensor. The hydrogen sensing characteristics are elevated the range of 10 50 ppm.

\section{Experimental}

$0.1 \mu \mathrm{m}$ solid carbon source of amorphous $3 \mathrm{C}-\mathrm{SiC}$ was deposited on $\mathrm{SiO}_{2}$ substrate by CVD at $1000{ }^{\circ} \mathrm{C}$. Transition metal of $200 \mathrm{~nm} \mathrm{Ni}$ was deposited by sputtering. Graphene synthesized on the $\mathrm{Ni}$ layer by annealing process under ramping speed, annealing time and cooling speed were $30{ }^{\circ} \mathrm{C} / \mathrm{s}, 3$ mins and $50{ }^{\circ} \mathrm{C} / \mathrm{s}$, respectively. Annealing temperature was kept at $1150{ }^{\circ} \mathrm{C}$. After annealing process,

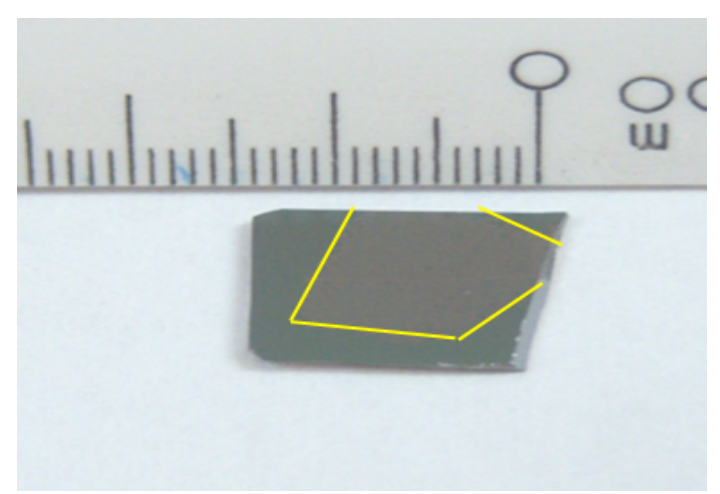

Fig. 1. Optical image of transferred graphene onto the $\mathrm{SiO}_{2}$ substrate. 
transferred onto the $\mathrm{SiO}_{2}$ substrate. Au electrode was formed on graphene by lift-off and I-V shows the $\mathrm{Au}$ and graphene is ohmic contact. Pd nano particle was deposited for hydrogen catalyst metal. Chemical bonding structure, response, recovery time and response factor were evaluated. Fig 1 shows transferred bare graphene onto the $\mathrm{SiO}_{2}$ substrate.

\section{Results and discussion}

Fig. 2 shows the Raman spectra of transferred graphene onto $\mathrm{SiO}_{2}$. The $\mathrm{I}_{\mathrm{G}} / \mathrm{I}_{\mathrm{D}}$ ratio was 2.73 and this value is higher than reported data [4]. $D$ and $G$ peaks are almost not changed with Raman measurement position. However, variation of $2 \mathrm{D}$ peak with each measurement position means the number of layer of graphene is different.

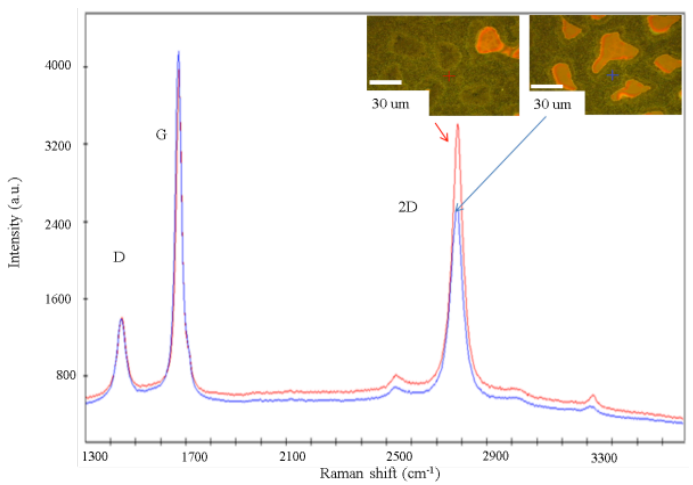

Fig. 2. Raman spectra of transferred graphene onto the $\mathrm{SiO}_{2}$ substrate.

Fig. 3 shows TEM image of transferred graphene. 6 carbon atoms combined with each other carbons. However, it shows not symmetry structure. This means the synthesized graphene have some strain and disorder. These defects can be reducing using other transfer method.

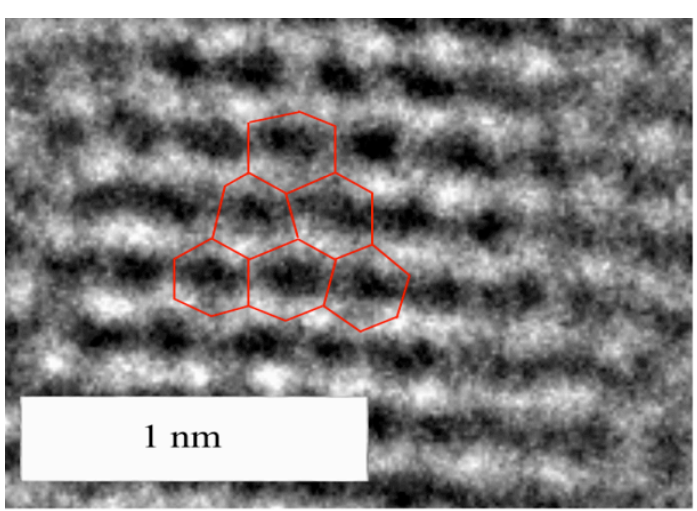

Fig. 3. TEM image of transferred graphene.

Fig. 4 shows the I-V curve of graphene and $\mathrm{Pd}$ doped graphene. Initial resistance of graphene was around of $360 \Omega$ and after $\mathrm{Pd}$ nano particle deposition, the resistance decreased to $27 \Omega$. It means the Pd nano particles increased the current flow passes way.

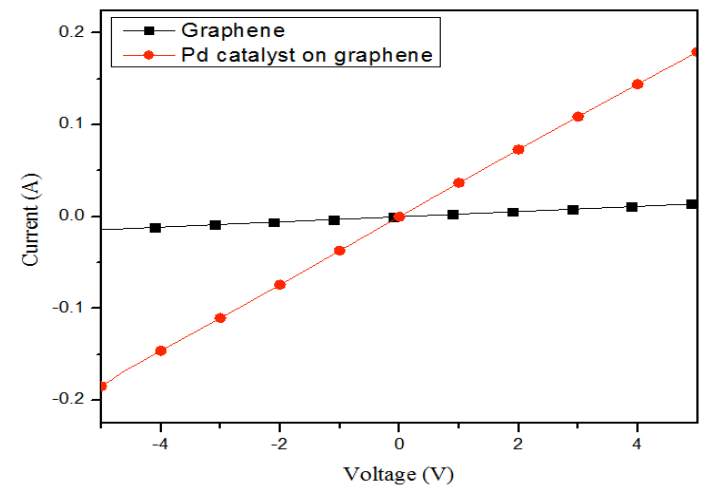

Fig. 4. I-V curve of graphene and Pd doped graphene.

The hydrogen sensing characteristics of graphene and $\mathrm{Pd}$ doped graphene are shown at Fig. 5 (a) and (b), respectively. The hydrogen concentration was varied in the range of 10 to $50 \mathrm{ppm}$. The resistance increased with the increase of hydrogen concentration.

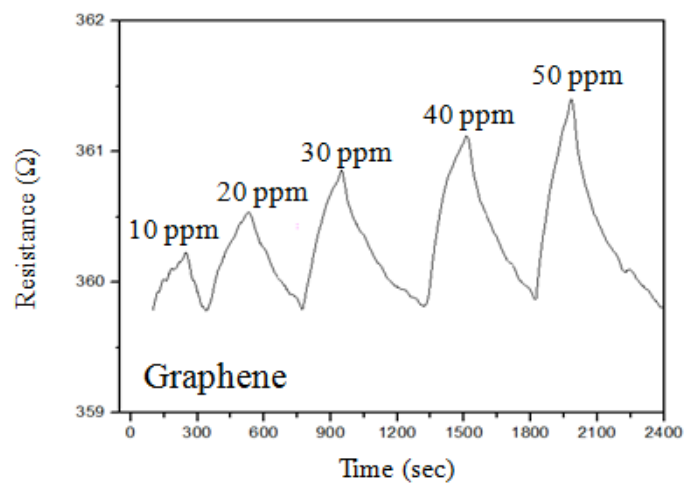

(a)

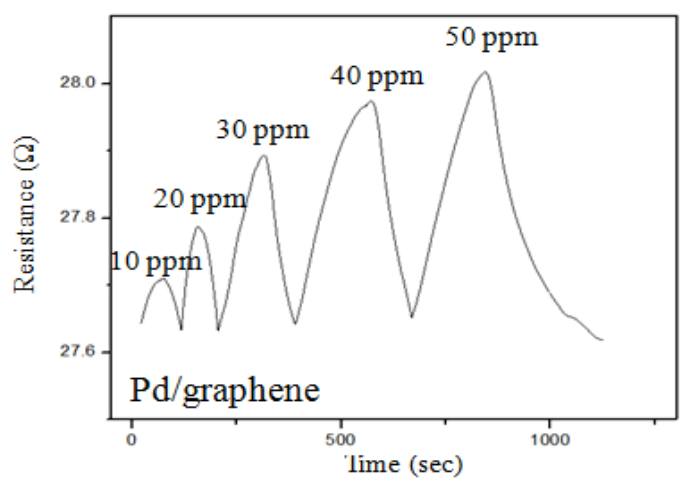

(b)

Fig. 5. Hydrogen sensing characteristics of (a) graphene and (b) Pd doped graphene.

Fig. 6 (a) and (b) shows response time (time to reach $90 \%$ of the maximum transition value) and the recovery time (time to reach $90 \%$ of the initial value) of graphene and $\mathrm{Pd}$ doped graphene, respectively. Response and recovery time increased with increase of hydrogen 
concentrations. At 50 ppm hydrogen concentration, response and recovery time of graphene was 190, $400 \mathrm{sec}$ and $\mathrm{Pd}$ doped graphene was 140, $250 \mathrm{sec}$, respectively. $\mathrm{Pd}$ catalyst improved reaction of graphene and hydrogen atom. These results are more faster performance compared with reported data $[5,6]$.

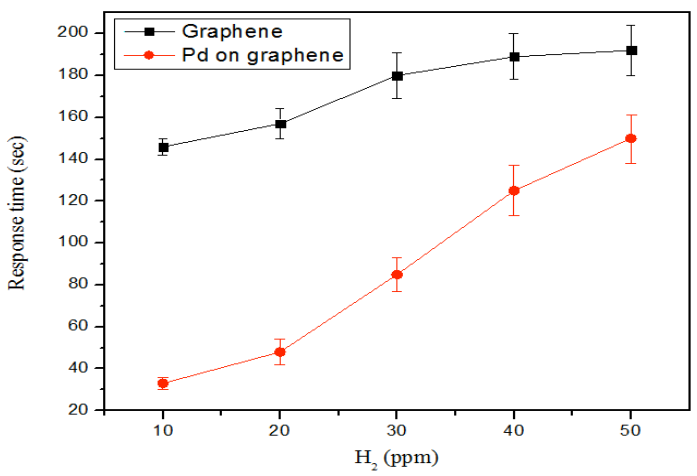

(a)

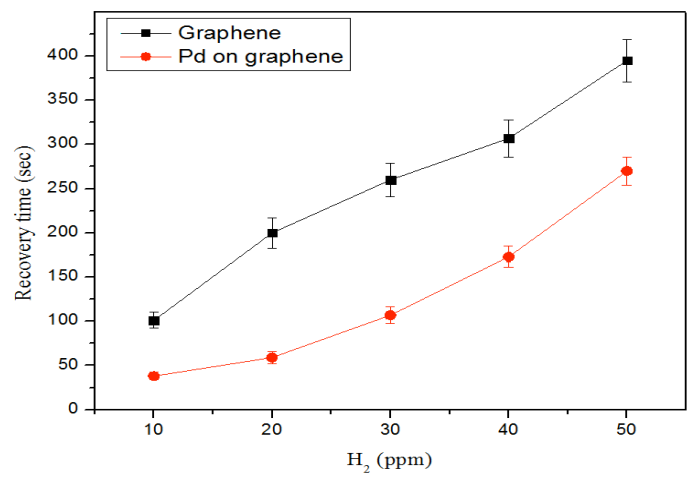

(b)

Fig. 6. Response and recovery time of graphene and $P d$ doped graphene with hydrogen concentration.

Tab. 1 shows response factors of graphene and $\mathrm{Pd}$ doped graphene, respectively. The response factor can be considered as an indirect measurement of the device sensor signal. The response factor was calculated as follows:

$$
\text { Response factor (\%) }=\left(\frac{R_{\text {initial }}-R_{\text {response }}}{R_{\text {initial }}}\right) \cdot 100
$$

where $R_{\text {initial }}$ is the resistance measured in air and $R_{\text {response }}$ is the resistance at each hydrogen concentrations. Response factor of graphene was 0.28 at 50 ppm. Pd doped graphene shows the higher response factor of 1.26 at 50 ppm.

\section{Conclusions}

In this work, graphene based hydrogen sensors were fabricated and evaluated. Graphene layer was achieved by annealing of $\mathrm{Ni} / 3 \mathrm{C}-\mathrm{SiC}$ structure and $\mathrm{Ni}$ etching in $\mathrm{HF}$ solution. Transferred graphene shows $I_{G} / I_{D}$ ratio of 2.73. By strain and disorder, 6 carbon atoms combine structure was not symmetry. Pd nano particle was used as catalyst and $\mathrm{Pd}$ doped graphene shows low resistance compared with bare graphene. 10 50 ppm hydrogen could be detected using graphene. However, response, recovery time and response factor improved using Pd catalyst. Sensors based on graphene are feasible for use in solid-state gas sensors designed for low concentration gas sensor.

Tab. 1: Response factor (\%) with hydrogen concentration.

\begin{tabular}{|c|c|c|}
\hline $\mathrm{H}_{2}(\mathrm{ppm})$ & graphene & Pd doped graphene \\
\hline 10 & 0.13 & 0.26 \\
\hline 20 & 0.19 & 0.53 \\
\hline 30 & 0.25 & 0.76 \\
\hline 40 & 0.27 & 0.91 \\
\hline 50 & 0.28 & 1.26 \\
\hline
\end{tabular}

\section{Acknowledgement}

This research was supported by the Korea Research Foundation Grant through the Human Resource Training Project for Regional Innovation funded by 2011 the Korean Government which was conducted by the Ministry of Education, Science and Technology.

\section{References}

[1] K.S. Novoselov, A.K. Geim, S.V. Morozov, D. Jiang, Y. Zhang, S.V. Dubonos, I.V. Grigorieva, A. A. Firsov, Electric Field Effect in Atomically Thin Carbon Films, Science 306, 666-669 (2004); doi: 10.1126/science.1102896

[2] E.H. Hwang, S. Adam, S.D. Sarma, Transport in chemically doped graphene in the presence of adsorbed molecules, Physical Review B 1, 1-6 (2006); doi: 10.1103/PhysRevB.76.195421

[3] D. Jena, A. Konar, Enhancement of Carrier Mobility in Semiconductor Nanostructures by Dielectric Engineering, Physical Review Letters 98, 136805(1)-136805(5) (2007); doi: 10.1103/ PhysRevLett.98.136805

[4] M.I. Kyu, L.J. Hyun, S. Rodney, L.H. Young, Reduced graphene oxide by chemical graphitization, Nature Communications 1, 1-6 (2010); doi: 10.1038/ncomms1067

[5] W. Wu, Z. Liu, L.A. Jauregui, Q. Yu, R. Pillai, H. Cao, J. Bao, Y.P. Chen, S.S. Pei, Wafer-scale synthesis of graphene by chemical vapor deposition and its application in hydrogen sensing, Sensors and Actuators B: Chemical 150, 296-300 (2010); doi: 10.1016/j.snb.2010.06.070

[6] B.H. Chu, J. Nicolosi, C.F. Lo, W. Strupinski, S.J. Pearton, F. Ren, Effect of Coated Platinum Thickness on Hydrogen Detection Sensitivity of Graphene-Based Sensors, Electrochemical and Solid-State Letters 14, K43-K45 (2011); doi: 10. 1149/ 1.3589250 